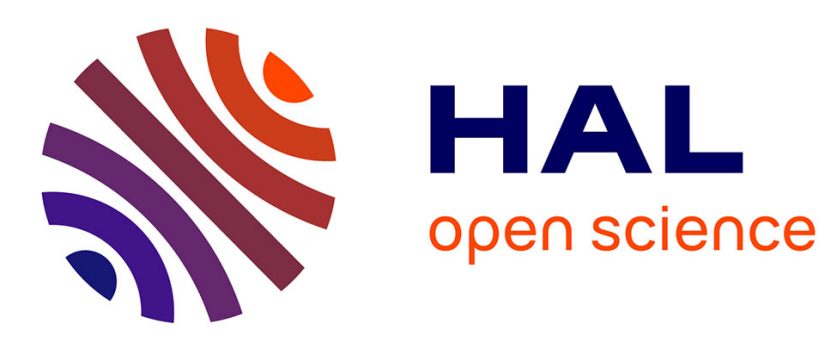

\title{
Polynomial complexity of solving systems of few algebraic equations with small degrees
}

Dima Grigoriev

\section{To cite this version:}

Dima Grigoriev. Polynomial complexity of solving systems of few algebraic equations with small degrees. Lecture Notes in Computer Science, 2013. hal-03044756

\section{HAL Id: hal-03044756 \\ https://hal.science/hal-03044756}

Submitted on 7 Dec 2020

HAL is a multi-disciplinary open access archive for the deposit and dissemination of scientific research documents, whether they are published or not. The documents may come from teaching and research institutions in France or abroad, or from public or private research centers.
L'archive ouverte pluridisciplinaire HAL, est destinée au dépôt et à la diffusion de documents scientifiques de niveau recherche, publiés ou non, émanant des établissements d'enseignement et de recherche français ou étrangers, des laboratoires publics ou privés. 


\title{
Polynomial complexity of solving systems of few algebraic equations with small degrees
}

\author{
Dima Grigoriev \\ CNRS, Mathématiques, Université de Lille \\ Villeneuve d'Ascq, 59655, France \\ Dmitry.Grigoryev@math .univ-lille1.fr \\ http://en.wikipedia.org/wiki/Dima_Grigoriev
}

\begin{abstract}
An algorithm is designed which tests solvability of a system of $k$ polynomial equations in $n$ variables with degrees $d$ within complexity polynomial in $n^{d^{3 k}}$. If a systems is solvable then the algorithm yields one of its solutions. Thus, for fixed $d, k$ the complexity of the algorithm is polynomial.
\end{abstract}

Keywords: polynomial complexity, solving systems of few equations with small degrees

\section{Introduction}

Consider a system of polynomial equations

$$
f_{1}=\cdots=f_{k}=0
$$

where $f_{1}, \ldots, f_{k} \in \mathbb{Z}\left[X_{1}, \ldots, X_{n}\right], \operatorname{deg} f_{i} \leq d, 1 \leq i \leq k$. The algorithm from [3], [1] (see also [2]) solves (1) within complexity polynomial in $M, k, d^{n^{2}}$, where $M$ denotes the bound on bit-sizes of (integer) coefficients of polynomials $f_{1}, \ldots, f_{k}$. Moreover, this algorithm finds the irreducible components of the variety in $\mathbb{C}^{n}$ determined by (1). We mention also that in [8] an algorithm is designed which tests solvability of (1) reducing it to a system of equations over $\mathbb{R}$, within a better complexity polynomial in $M,(k \cdot d)^{n}$. We note that the algorithm from [8] tests solvability of (1) and outputs a solution, provided that (1) is solvable, rather than finds the irreducible components as the algorithms from [3], [1].

In the present paper we design an algorithm which tests solvability of (1) within complexity polynomial in $M \cdot\left(\begin{array}{c}n+d^{3 k} \\ n\end{array}\right) \leq M \cdot n^{d^{3 k}}$, which provides polynomial (in the size $M \cdot k \cdot\left(\begin{array}{c}n+d \\ n\end{array}\right)$ of the input system (1)) complexity when $d, k$ being fixed. If (1) is solvable then the algorithm yields one of its solutions. Note that the algorithm from [8] has a 
polynomial complexity when, say $d>n^{2}$ and $k$ being polynomial in $n$; when $d$ is close to $n$ the complexity is subexponential, while for small $d$ the complexity is exponential.

We mention that in [6] an algorithm was designed testing solvability of (1) over $\mathbb{R}$ (and finding a real solution, provided that it does exist) within the complexity polynomial in $M, n^{2 k}$ for quadratic equations $(d=2)$, and moreover, one can replace equations by inequalities.

It would be interesting to clarify, for which relations between $n, k, d$ the complexity of solvability of (1) is polynomial. In particular, when $d=2$ and $k$ is close to $n$ the problem of solvability is $N P$-hard.

\section{Testing points for sparse polynomials}

Recall (see [4]) a construction of testing points for sparse polynomials in $n$ variables. Let $p_{i}$ denote $i$-th prime and $s_{j}=\left(p_{1}^{j}, \ldots, p_{n}^{j}\right) \in \mathbb{Z}^{n}, j \geq 0$ be a point. A polynomial $f \in \mathbb{C}\left[X_{1}, \ldots, X_{n}\right]$ is called $t$-sparse if it contains at most $t$ monomials.

Lemma 1.1 [4]. For a t-sparse polynomial $f$ there exists $0 \leq j<t$ such that $f\left(s_{j}\right) \neq 0$.

The proof follows from the observation that writing $f=\sum_{1 \leq l \leq t} a_{l} \cdot X^{I_{l}}$ where coefficients $a_{l} \in \mathbb{C}$ and $X^{I_{l}}$ are monomials, the equations $f\left(s_{j}\right)=0,0 \leq j<t$ lead to a $t \times t$ linear system with Vandermonde matrix and its solution $\left(a_{1}, \ldots, a_{t}\right)$. Since Vandermonde matrix is nonsingular, the obtained contradiction proves the lemma.

Corollary 1.2 Let $\operatorname{deg} f \leq D$. There exists $0 \leq j<\left(\begin{array}{c}n+D \\ n\end{array}\right)$ such that $f\left(s_{j}\right) \neq 0$.

\section{Reduction of solvability to systems in few variables}

The goal of this section is to reduce testing solvability of (1) to testing solvability of several systems in $k$ variables.

Let $V \subset \mathbb{C}^{n}$ be an irreducible (over $\mathbb{Q}$ ) component of the variety determined by (1). Observe that the algorithm described in the next Section does not need to produce $V$. Then $\operatorname{dim} V=: m \geq n-k$ and $\operatorname{deg} V \leq d^{n-m} \leq d^{k}$ due to Bezout inequality [9].

Let variables $X_{i_{1}}, \ldots, X_{i_{m}}$ constitute a transcendental basis over $\mathbb{C}$ of the field $\mathbb{C}(V)$ of rational functions on $V$, clearly such $i_{1}, \ldots, i_{m}$ do exist. Then the degree of fields extension $e:=\left[\mathbb{C}(V): \mathbb{C}\left(X_{i_{1}}, \ldots, X_{i_{m}}\right)\right] \leq \operatorname{deg} V$ equals the typical (and at the same time, the maximal) number of points in the intersections $V \cap\left\{X_{i_{1}}=c_{1}, \ldots, X_{i_{m}}=c_{m}\right\}$ for different $c_{1}, \ldots, c_{m} \in \mathbb{C}$, provided that this intersection being finite. Observe that for almost all vectors $\left(c_{1}, \ldots, c_{m}\right) \in \mathbb{C}^{n}$ the intersection is finite and consists of $e$ points.

There exists a primitive element $Y=\sum_{i \neq i_{1}, \ldots, i_{m}} b_{i} \cdot X_{i}$ of the extension $\mathbb{C}(V)$ of the field $\mathbb{C}\left(X_{i_{1}}, \ldots, X_{i_{m}}\right)$ for appropriate integers $b_{i}[7]$ (moreover, one can take integers $0 \leq b_{i} \leq e$ for all $i$, see e. g. [1], [3], but we do not need here these bounds). Moreover, there exist 
$n-m$ linearly over $\mathbb{C}$ independent primitive elements $Y_{1}, \ldots, Y_{n-m}$ of this form. One can view $Y_{1}, \ldots, Y_{n-m}, X_{i_{1}}, \ldots, X_{i_{m}}$ as new coordinates.

Consider a linear projection $\pi_{l}: \mathbb{C}^{n} \rightarrow \mathbb{C}^{m+1}$ onto the coordinates $Y_{l}, X_{i_{1}}, \ldots, X_{i_{m}}, 1 \leq$ $l \leq n-m$. Then the closure $\overline{\pi_{l}(V)} \subset \mathbb{C}^{m+1}$ is an irreducible hypersurface, so $\operatorname{dim} \overline{\pi_{l}(V)}=$ $m$. Denote by $g_{l} \in \mathbb{Q}\left[Y_{l}, X_{i_{1}}, \ldots, X_{i_{m}}\right]$ the minimal polynomial providing the equation of $\overline{\pi_{l}(V)}$. Then $\operatorname{deg} g_{l}=\operatorname{deg} \overline{\pi_{l}(V)} \leq \operatorname{deg} V[9]$ and $\operatorname{deg}_{Y_{l}} g_{l}=e$, taking into account that $Y_{l}$ is a primitive element.

Rewriting $g_{l}=\sum_{q \leq e} Y_{l}^{q} \cdot h_{q}, h_{q} \in \mathbb{Q}\left[X_{i_{1}}, \ldots, X_{i_{m}}\right]$ as a polynomial in a distinguished variable $Y_{l}$, we denote $H_{l}:=h_{e} \cdot \operatorname{Disc}_{Y_{l}}\left(g_{l}\right) \in \mathbb{Q}\left[X_{i_{1}}, \ldots, X_{i_{m}}\right]$, where $\operatorname{Disc}_{Y_{l}}$ denotes the discriminant with respect to the variable $Y_{l}$ (the discriminant does not vanish identically since $Y_{l}$ is a primitive element). We have $\operatorname{deg} H_{l} \leq d^{k}+d^{2 k}$. Consider the product $H:=\prod_{1 \leq l \leq n-m} H_{l}$, then $D:=\operatorname{deg} H \leq(n-m) \cdot\left(d^{k}+d^{2 k}\right) \leq d^{3 k}$.

Due to Corollary 1.2 there exists $0 \leq j<\left(\begin{array}{c}D+m \\ D\end{array}\right) \leq m^{d^{3 k}}$ such that $H\left(s_{j}\right)=$ $H\left(p_{1}^{j}, \ldots, p_{m}^{j}\right) \neq 0$. Observe that the projective intersection $\bar{V} \cap\left\{X_{i_{1}}=p_{1}^{j} \cdot X_{0}, \cdots, X_{i_{m}}=\right.$ $\left.p_{m}^{j} \cdot X_{0}\right\}$ in the projective space $\mathbb{P} \mathbb{C}^{n} \supset \mathbb{C}^{n}$ with the coordinates $\left[X_{0}: X_{1}: \cdots: X_{n}\right]$ consists of $e$ points, where $\bar{V}$ denotes the projective closure of $V$. On the other hand, coordinate $Y_{l}$ of the points of the affine intersection $V \cap\left\{X_{i_{1}}=p_{1}^{j}, \ldots, X_{i_{m}}=p_{m}^{j}\right\}$ attains $e$ different values, taking into account that $H_{l}\left(s_{j}\right) \neq 0,1 \leq l \leq n-m$. Therefore, all $e$ points from the projective intersection lie in the affine chart $\mathbb{C}^{n}$. Consequently, the intersection $V \cap\left\{X_{i_{1}}=p_{1}^{j}, \ldots, X_{i_{m}}=p_{m}^{j}\right\}$ is not empty.

\section{Test of solvability and its complexity}

Thus, to test solvability of (1) the algorithm chooses all possible subsets $\left\{i_{1}, \ldots, i_{m}\right\} \subset$ $\{1, \ldots, n\}$ with $m \geq n-k$ treating $X_{i_{1}}, \ldots, X_{i_{m}}$ as a candidate for a transcendental basis of some irreducible component $V$ of the variety determined by (1). After that for each $0 \leq j<\left(\begin{array}{c}D+m \\ D\end{array}\right)$ where $D \leq d^{3 k}$, the algorithm substitutes $X_{i_{1}}=p_{1}^{j}, \ldots, X_{i_{m}}=p_{m}^{j}$ into polynomials $f_{1}, \ldots, f_{k}$ and solves the resulting system of polynomial equations in $n-m \leq k$ variables applying the algorithm from [1], [3]. The complexity of each of these applications does not exceed a polynomial in $M \cdot\left(\begin{array}{c}D+m \\ D\end{array}\right) \cdot d^{(n-m)^{2}}$, i. e. a polynomial in $M \cdot n^{d^{3 k}}$. Moreover, the algorithm from [1], [3] yields a solution of a system, provided that it does exist. Summarizing, we obtain the following theorem.

Theorem 3.1 One can test solvability over $\mathbb{C}$ of a system (1) of $k$ polynomials $f_{1}, \ldots, f_{k} \in$ $\mathbb{Z}\left[X_{1}, \ldots, X_{n}\right]$ with degrees $d$ within complexity polynomial in $M \cdot\left(\begin{array}{c}n+d^{3 k} \\ n\end{array}\right) \leq M \cdot n^{d^{3 k}}$, where $M$ bounds the bit-sizes of (integer) coefficients of $f_{1}, \ldots, f_{k}$. If (1) is solvable then the algorithm yields one of its solutions.

Corollary 3.2 For fixed $d, k$ the complexity of the algorithm is polynomial.

The construction and the Theorem extend literally to polynomials with coefficients from a field $F$ of characteristic zero (for complexity bounds one needs that the elements of 
$F$ are given in an efficient way). For $F$ of a positive characteristic one can obtain similar results replacing the zero test from Section 1 by the zero test from [5].

Acknowledgements. The author is grateful to the Max-Planck Institut für Mathematik, Bonn for its hospitality during writing this paper.

\section{References}

[1] A. Chistov, An algorithm of polynomial complexity for factoring polynomials, and determination of the components of a variety in a subexponential time, J.Soviet Math., 34 (1986), 1838-1882.

[2] A. Chistov, D. Grigoriev, Complexity of quantifier elimination in the theory of algebraically closed fields, Lect. Notes Comput. Sci., 176 (1984), 17-31.

[3] D. Grigoriev, Polynomial factoring over a finite field and solving systems of algebraic equations, J. Soviet Math., 34 (1986), 1762-1803.

[4] D. Grigoriev, M. Karpinski, The matching problem for bipartite graphs with polynomially bounded permanents is in NC, Proc. 28 Symp. Found. Comput. Sci., IEEE, (1987), 166-172.

[5] D. Grigoriev, M. Karpinski, M. Singer, Fast parallel algorithms for sparse multivariate polynomial interpolation over finite fields, SIAM J. Comput., 19 (1990), 1059-1063.

[6] D. Grigoriev, D. Pasechnik, Polynomial-time computing over quadratic maps I. Sampling in real algebraic sets, Computational Complexity, 14 (2005), 20-52.

[7] S. Lang, Algebra, Springer, 2002.

[8] J. Renegar, On the computational complexity and geometry of the first-order theory of the reals. I. Introduction. Preliminaries. The geometry of semi-algebraic sets. The decision problem for the existential theory of the reals, J. Symbolic Comput. 13 (1992), 255-299.

[9] I. Shafarevich, Foundations of algebraic geometry, MacMillan Journals, 1969. 